\title{
Estimação de Canal Melhorada Baseada em Projeção em Subespaço para Sistemas MC-CDMA
}

\author{
Deolinda Fontes Cardoso, Fabian David Backx, Raimundo Sampaio Neto
}

\begin{abstract}
Resumo-Este trabalho analisa um procedimento simples para melhorar a qualidade da estimativa da resposta em frequência do canal em sistemas MC-CDMA-CP. A análise considera o enlace reverso e os canais são seletivos em frequência. A melhoria é obtida por meio de uma matriz pré-computada que projeta a estimativa original da resposta em frequência do canal no subespaço onde a verdadeira resposta em frequência está contida. Usando a formulação de matriz de projeção, expressões analíticas e limitantes, independentes das características do canal, são derivados para o erro médio quadrático normalizado dos estimadores de canal. Extensivas simulações demonstraram que os estimadores de canal melhorados têm grande impacto no desempenho do sistema.
\end{abstract}

Palavras-Chave-Estimação de Canal Assistida, Matriz de Projeção, MC-CDMA-CP.

Abstract-This paper considers an improved pilot-aided channel estimation scheme to reduce the multi access interference in MC-CDMA systems. The improved channel estimator is implemented through a projection matrix that refines the original channel estimator. Analytical expressions for the mean square error and its channel independent upper and lower bounds are derived for both the original and improved estimators for cyclicprefixed MC-CDMA uplink transmissions in the presence of frequency-selective channels. Computer simulations illustrate the impact of the improved channel estimator on MC-CDMA system performance.

Keywords-Pilot-aided Channel Estimation, Projection Matrix, MC-CDMA-CP.

\section{INTRODUÇÃO}

A engenhosa associação da técnica DS-CDMA [1] e modulação OFDM [2] originou os sistemas CDMA com múltiplas portadoras (MC-CDMA) [3], nos quais os símbolos de dados de cada usuário são espalhados por toda a banda disponível e os chips são simultaneamente transmitidos um sobre cada subportadora. Uma estratégia para mitigar a interferência entre blocos (IEB), emprestada de OFDM, consiste em antes da transmissão inserir no início de cada bloco de símbolos um intervalo de guarda suficiente para acomodar as parcelas do multipercurso do canal. O intervalo de guarda considerado é do tipo de prefixo cíclico CP (Cyclic Prefix) [4]. No caso de detecção coerente, faz-se necessário estimar, na estação receptora, o canal de comunicação. Neste estudo, consideramos a estimação do canal assistida por símbolos piloto e, assim, o objetivo do trabalho é analisar um procedimento simples e eficiente para melhorar a qualidade

Centro de Estudos em Telecomunicações (CETUC), Pontifícia Universidade Católica do Rio de Janeiro (PUC-Rio), Brasil, E-mails: \{deolinda,fdb,raimundo\}@cetuc.puc-rio.br. Este trabalho foi parcialmente financiado pelo Centro de Análises de Sistemas Navais (CASNAV-MB) e Conselho Nacional de Pesquisa e Desenvolvimento (CNPq). dos estimadores de canal convencionais baseados no critério MMSE (Minimum Mean Square Error). Estimadores de canal já são bem conhecidos na literatura para sistemas CDMA [5] e, também, já apresentados para modulações OFDM [6]. Para o sistema MC-CDMA, foco deste trabalho, a novidade consiste em, partindo de [6], explorar um novo enfoque àquela idéia por meio de uma matriz de projeção que projeta a estimativa de canal no subespaço onde a verdadeira resposta de frequência do equivalente discreto do canal está contida [7], [8], [9]. São apresentadas análises estatísticas e expressões para limitantes do erro médio quadrático dos estimadores, que independem das características do canal. Tais expressões podem ser usadas no projeto de um sistema de forma a selecionar, a partir de um determinado conjunto de assinaturas, um subconjunto ótimo para ser designado aos usuários do sistema. Esse subconjunto garante a minimização da parcela decorrente da Interferência de Múltiplo Acesso (IMA) presente na expressão do erro médio quadrático dos estimadores de canal. Por fim, são apresentadas expressões dos fatores de redução da IMA e do ruído decorrentes da melhoria proposta nos estimadores. Os resultados analíticos e obtidos por simulação indicaram um desempenho satisfatório dos estimadores melhorados propostos, principalmente quando o sistema MC-CDMA opera com carga elevada. Este trabalho está organizado da seguinte forma: a Seção II descreve o modelo vetorial dos sinais MC-CDMA; a Seção III apresenta as análises de estimação assistida; a Seção IV apresenta a equalização e detecção; a Seção V descreve os resultados numéricos; e finalmente na Seção VI apresentamos as conclusões do trabalho. Notação: caracteres maiúsculos em negrito denotam matrizes; caracteres minúsculos em negrito denotam vetores. Os operadores $(.)^{T},(.)^{H}$ e $(.)^{*}$ indicam transposto de uma matriz, hermitiano de um vetor, e conjugado de um escalar complexo; $(\cdot)^{-1}$ representa a inversa de uma matriz. O operador traço da matriz $\mathbf{A}$ é $\operatorname{tr}(\mathbf{A})$. O operador $E[\cdot]$ representa o valor médio e $\mathbf{I}_{M}$ denota uma matriz identidade de dimensões $M \times M$.

\section{O Modelo Vetorial dos Sinais MC-CDMA}

Para o enlace reverso de uma célula com $K$ usuários ativos são consideradas transmissões síncronas em $M$ subportadoras e os símbolos transmitidos são oriundos de constelações bidimensionais com módulo constante. Considera-se que a resposta ao impulso do canal permanece constante durante a transmissão de cada símbolo. No transmissor o fluxo de dados original é espalhado por uma seqüência de espalhamento (código) de comprimento $M$, cujos chips são convertidos de serial para paralelo formando blocos de dimensão $M$ aos 
quais é aplicada a transformada de Fourier inversa normalizada (IDFT) de dimensões $M \times M$, representada pela matriz $\mathbf{F}_{M, M}^{H}$, sendo $\mathbf{F}_{M, M}^{H} \mathbf{F}_{M, M}=\mathbf{F}_{M, M} \mathbf{F}_{M, M}^{H}=\mathbf{I}_{M, M}$. Com o intuito de evitar a IEB (na recepção) um intervalo de guarda do tipo $\mathrm{CP}$ de comprimento $G$ é inserido ao início de cada bloco. O novo bloco resultante, de dimensão $P=M+G$, é transmitido através do canal multipercurso. No receptor o sinal é processado por um filtro casado ao pulso de chip e amostrado a taxa de chip. Assim, o equivalente discreto do canal é aqui modelado como um filtro FIR com $L$ coeficientes cujos ganhos são amostras tomadas a taxa de chips da resposta ao impulso do canal associado a cada usuário: $\mathbf{h}_{k}=\left[\begin{array}{lll}h_{k_{0}} & \ldots & h_{k_{L-1}}\end{array}\right]^{T}$. Além disso, considera-se que todos os canais apresentam o mesmo comprimento $L$ e este não excede o intervalo de guarda $G(G \geq L)$.

O enlace de transmissão do canal multipercurso, entre a estação do $k$-ésimo usuário e a Estação Rádio Base, pode ser representado por uma matriz de convolução Toeplitz triangular inferior $\mathbf{H}_{k}$ de dimensões $P \times P$, com a primeira coluna: $\left[\begin{array}{llllll}h_{k_{0}} & \ldots & h_{k_{L-1}} & 0 & \ldots & 0\end{array}\right]^{T}$. O procedimento seguinte no receptor consiste em remover o intervalo de guarda $\mathrm{CP}$ do bloco recebido e aplicar a transformada direta de Fourier (DFT) de dimensões $M \times M$ o que permite transformar a matriz de convolução do canal em uma matriz diagonal. Assim, o agregado de sinais recebidos na estação base é expresso por:

$$
\mathbf{r}(i)=\sum_{k=1}^{K} \mathbf{Q}_{k} \mathbf{c}_{k} b_{k}(i)+\mathbf{n}(i)=\sum_{k=1}^{K} \mathbf{C}_{k} \mathbf{q}_{k} b_{k}(i)+\mathbf{n}(i),
$$

onde $\mathbf{C}_{k}=\operatorname{diag}\left(\mathbf{c}_{k}\right)$ é uma matriz diagonal contendo os chips da sequência de espalhamento do $k$-ésimo usuário $\mathbf{c}_{k}=$ $\left[c_{k, 0}, \ldots c_{k, M-1}\right]^{T}$ com módulo constante $\left|c_{k, j}\right|^{2}=1 / M$, $\forall j$; o vetor complexo de ruído aditivo branco é representado por $\mathbf{n}(i)=\left[\begin{array}{lll}n_{0}(i) & \ldots & n_{N-1}(i)\end{array}\right]^{T}$ cuja matriz de covariância é $\mathrm{E}\left[\mathbf{n}(i) \mathbf{n}^{H}(i)\right]=\sigma^{2} \mathbf{I}_{M}$ e $b_{k}(i)$ é o símbolo transmitido pelo $k$-ésimo usuário com $\left|b_{k}(i)\right|=1$ e $\mathrm{E}\left[b_{k}(i)\right]=0$, sendo que símbolos transmitidos por diferentes usuários são estatisticamente independentes. A matriz do canal é dada por $\mathbf{Q}_{k}=\operatorname{diag}\left(\mathbf{q}_{k}\right)$ onde o vetor da resposta em freqüência do canal do $k$-ésimo usuário é dado por:

$$
\mathbf{q}_{k}=\sqrt{M} \mathbf{F}_{M, M} \mathbf{h}_{k_{e x t}}
$$

onde $\mathbf{h}_{k_{e x t}}=\left[h_{k_{0}} \ldots h_{k_{L-1}} 0 \ldots 0\right]$ é a resposta ao impulso do canal, de comprimento $L$, estendida com $M-L$ zeros. Observe que o vetor da resposta em frequência do canal pode, também, ser escrito em função da resposta ao impulso do canal sem a extensão de zeros:

$$
\mathbf{q}_{k}=\sqrt{M} \mathbf{F}_{M, L} \mathbf{h}_{k}
$$

onde $\mathbf{F}_{M, L}$ é uma matriz contendo as primeiras $L$ colunas da matriz de Fourier $\mathbf{F}_{M, M}$ de dimensões $M \times M$.

\section{EstimaÇÃO DE CANAL}

A estimativa do canal associada ao $m$-ésimo usuário de interesse, é baseada em [5] e corresponde ao critério de mínimo erro médio quadrático.

$$
\widehat{\mathbf{q}}_{m}^{(m m s e)}=\arg \min _{\mathbf{q}} \mathbf{E}\left[\left\|\mathbf{r}(i)-\mathbf{C}_{m} \mathbf{q} b_{m}(i)\right\|^{2}\right],
$$

onde $b_{m}(i)$ representa o símbolo piloto transmitido pelo $m$ ésimo usuário para estimação da resposta em freqüência de seu canal de transmissão $\mathbf{q}_{m}$. A solução da expressão (4) resulta em $\widehat{\mathbf{q}}_{m}^{(m m s e)}=\mathbf{C}_{m}^{-1} \mathbf{E}\left[\mathbf{r}(i) b_{m}^{*}(i)\right]=\mathbf{q}_{m}$. Aproximando o valor esperado por uma média envolvendo os $N_{p}$ símbolos piloto transmitidos, obtém-se o estimador despolarizado dado por:

$$
\widehat{\mathbf{q}}_{m}=\frac{\mathbf{C}_{m}^{-1}}{N_{p}} \sum_{i=1}^{N_{p}} \mathbf{r}(i) b_{m}^{*}(i) .
$$

\section{A. Estimativa de Canal Melhorada}

Com o objetivo de refinar a estimativa dada em (5) pode ser aplicada uma melhoria baseada na observação de que o comprimento da resposta ao impulso do canal é $L \leq M$. Como resultado, qualquer componente que esteja fora desse intervalo em uma dada estimativa da resposta ao impulso do canal é considerada ruidosa (não-significativa) e pode ser removida [6]. A obtenção da estimativa melhorada consiste assim, em aplicar na estimativa no domínio da freqüência $\widehat{\mathbf{q}}_{m}$, a IDFT de $M$-pontos. Uma vez obtido o vetor da estimativa da resposta ao impulso do canal $\widehat{\mathbf{h}}$, os seus $(M-L)$ últimos coeficientes são substituídos pelo valor zero. Desde que o interesse é obter a resposta em frequência do canal, as componentes significativas são transformadas para o domínio da frequência aplicando-se a DFT de $M$-pontos que retorna a estimativa para o domínio da freqüência, dada pelo vetor $\widehat{\mathbf{q}}_{m}^{w}$.

Sob outra perspectiva, pode ser mostrado [7] que o procedimento descrito corresponde à projeção da estimativa da resposta em frequiência do canal $\widehat{\mathbf{q}}_{m}$ no subespaço gerado pelas primeiras $L$ colunas da Matriz de Fourier $\mathbf{F}_{M, M}$. Note que devido a (3), a verdadeira resposta em freqüência tem que pertencer a esse subespaço. $\mathrm{O}$ valor correto do comprimento $L$ do canal pode não ser conhecido a priori, porém, como $L \leq G \leq M$ a verdadeira resposta em freqüência pertence também ao subespaço gerado pelas primeiras $G$ colunas de $\mathbf{F}_{M, M}$. A matriz $\mathbf{W}_{o}$ que projeta um vetor M-dimensional nesse subespaço é dada por:

$$
\mathbf{W}_{o}=\mathbf{F}_{M, G}\left(\mathbf{F}_{M, G}^{H} \mathbf{F}_{M, G}\right)^{-1} \mathbf{F}_{M, G}^{H},
$$

onde $\mathbf{F}_{M, G}$ é uma matriz $M \times G$ contendo as primeiras $G$ colunas da matriz de Fourier de $M$-pontos. Usando o fato de que $\mathbf{F}_{M, G}^{H} \mathbf{F}_{M, G}=\mathbf{I}_{G}$, (6) se reduz a $\mathbf{W}_{o}=\mathbf{F}_{M, G} \mathbf{F}_{M, G}^{H}$. Este processamento permite remover as componentes ruidosas não significativas da estimativa convencional, melhorando a qualidade da estimativa de canal resultante do procedimento [7]. Aplicando a matriz de projeção $\mathbf{W}_{o}$ na estimativa convencional, obtém-se:

$$
\widehat{\mathbf{q}}_{m}^{w}=\mathbf{W}_{o} \widehat{\mathbf{q}}_{m}=\mathbf{W}_{o} \mathbf{C}_{m}^{-1} \frac{1}{N_{p}} \sum_{i=1}^{N_{p}} \mathbf{r}(i) b_{m}^{*}(i) .
$$

Ressalta-se que independentemente de como a estimativa original foi obtida, a aplicação da matriz de projeção $\mathbf{W}_{o}$ resulta em uma redução no erro da estimativa (ver Apêndice). Contudo o ganho de desempenho é diferente para a componente do ruído aditivo e para a componente da IMA presentes no erro da estimativa de canal. 


\section{B. Desempenho dos Estimadores}

Para permitir uma formulação geral, define-se a matriz $\mathbf{A}_{m}$, de dimensões $M \times M$, que será utilizada posteriormente em dois casos distintos: 1) $\mathbf{A}_{m}=\mathbf{C}_{m}^{-1}$ em (5) e 2) $\mathbf{A}_{m}=\mathbf{W}_{o} \mathbf{C}_{m}^{-1}$ em (7); ambas conduzindo a estimadores despolarizados uma vez que $\mathbf{A}_{m} \mathbf{C}_{m} \mathbf{q}_{m}=\mathbf{q}_{m}$. A estimativa do canal do $m$-ésimo usuário pode ser escrita, separando o termo referente ao usuário de interesse da seguinte forma:

$\widehat{\mathbf{q}}_{m}=\mathbf{q}_{m}+\mathbf{A}_{m} \frac{1}{N_{p}} \sum_{k \neq m}^{K} \mathbf{C}_{k} \mathbf{q}_{k} \mathbf{b}_{m}^{H} \mathbf{b}_{k}+\mathbf{A}_{m} \frac{1}{N_{p}} \sum_{i=1}^{N_{p}} \mathbf{n}(i) b_{m}^{*}(i)$,

onde usou-se $\sum_{i=1}^{N_{p}} b_{m}^{*}(i) b_{k}(i)=\mathbf{b}_{m}^{H} \mathbf{b}_{k}$, sendo o vetor $\mathbf{b}_{m}=\left[b_{m}(1) \ldots b_{m}\left(N_{p}\right)\right]^{T}$. O erro médio quadrático da estimativa de canal para o $m$-ésimo usuário, condicionado ao conhecimento dos $K$ canais associados a cada usuário, é definido como:

$$
\varepsilon_{m}^{2}=\mathrm{E}\left[\left\|\widehat{\mathbf{q}}_{m}-\mathbf{q}_{m}\right\|^{2} \mid \mathbf{q}_{1}, \mathbf{q}_{2}, \ldots, \mathbf{q}_{K}\right] .
$$

Desenvolvendo (8), tem-se:

$$
\begin{aligned}
\varepsilon_{m}^{2} & =\frac{1}{N_{p}^{2}} \sum_{k \neq m}^{K} \sum_{t \neq m}^{K} \mathbf{q}_{k}^{H} \mathbf{C}_{k}^{H}\left(\mathbf{A}_{m}^{H} \mathbf{A}_{m}\right) \mathbf{C}_{t} \mathbf{q}_{t} \mathrm{E}\left[\mathbf{b}_{k}^{H} \mathbf{b}_{m} \mathbf{b}_{m}^{H} \mathbf{b}_{t}\right] \\
& +\frac{\sigma^{2}}{N_{p}} \operatorname{tr}\left[\mathbf{A}_{m} \mathbf{A}_{m}^{H}\right]
\end{aligned}
$$

onde o termo do valor esperado reduz-se a:

$$
\mathrm{E}\left[\mathbf{b}_{m}^{H} \mathbf{b}_{k} \mathbf{b}_{t}^{H} \mathbf{b}_{m}\right]=\left\{\begin{array}{ccc}
N_{p}, & \text { se } & k=t \\
0, & \text { se } & k \neq t
\end{array},\right.
$$

uma vez que, neste estudo, é considerado que os símbolos de usuários distintos são estatisticamente independentes. Chegase portanto a:

$$
\varepsilon_{m}^{2}=\frac{1}{N_{p}} \sum_{k=1, k \neq m}^{K} \mathbf{q}_{k}^{H} \mathbf{C}_{k}^{H} \mathbf{A}_{m}^{H} \mathbf{A}_{m} \mathbf{C}_{k} \mathbf{q}_{k}+\frac{\sigma^{2}}{N_{p}} \operatorname{tr}\left[\mathbf{A}_{m} \mathbf{A}_{m}^{H}\right] .
$$

Ou ainda:

$$
\begin{aligned}
\varepsilon_{m}^{2} & =\frac{1}{N_{p}}[\sum_{k=1}^{K} \mathbf{q}_{k}^{H} \mathbf{C}_{k}^{H} \mathbf{A}_{m}^{H} \mathbf{A}_{m} \mathbf{C}_{k} \mathbf{q}_{k}-\underbrace{\mathbf{q}_{m}^{H} \mathbf{C}_{m}^{H} \mathbf{A}_{m}^{H} \mathbf{A}_{m} \mathbf{C}_{m} \mathbf{q}_{m}}_{\left\|\mathbf{q}_{m}\right\|^{2}}] \\
& +\frac{\sigma^{2}}{N_{p}} \operatorname{tr}\left[\mathbf{A}_{m} \mathbf{A}_{m}^{H}\right] .
\end{aligned}
$$

A média do erro médio quadrático ao longo dos $K$ usuários é:

$$
\begin{aligned}
\varepsilon^{2} & =\frac{1}{K} \sum_{m=1}^{K} \varepsilon_{m}^{2} \\
& =\left[\frac{1}{N_{p}} \sum_{k=1}^{K} \mathbf{q}_{k}^{H} \mathbf{C}_{k}^{H} \mathbf{B} \mathbf{C}_{k} \mathbf{q}_{k}-\frac{1}{K} \sum_{m=1}^{K}\left\|\mathbf{q}_{m}\right\|^{2}\right]+\frac{\sigma^{2}}{N_{p}} \operatorname{tr}(\mathbf{B})(10)
\end{aligned}
$$

sendo

$$
\mathbf{B}=\frac{1}{K} \sum_{m=1}^{K} \mathbf{A}_{m} \mathbf{A}_{m}^{H}
$$

Para o procedimento a seguir, é suposto que os canais dos diferentes usuários são: $i)$ normalizados com $E\left[\left\|\mathbf{h}_{k}\right\|^{2}\right]=1 \mathrm{e}$, portanto, com $E\left[\left\|\mathbf{q}_{k}\right\|^{2}\right]=M$, e $\left.i i\right)$ identicamente distribuídos. Em consequência, podem ser usadas as estatísticas da resposta em frequência do canal de apenas um usuário; por exemplo, do primeiro usuário. Aplicando o valor esperado em (10), isto é $\epsilon^{2}=\mathrm{E}\left[\varepsilon^{2}\right]$, obtém-se:

$$
\epsilon^{2}=\frac{1}{N_{p}}\left[\mathrm{E}\left[\mathbf{q}_{1}^{H} \mathbf{D}_{o} \mathbf{q}_{1}\right]-M\right]+\frac{\sigma^{2}}{N_{p}} \operatorname{tr}(\mathbf{B})
$$

onde foi definida a matriz: $\mathbf{D}_{o}=\sum_{k=1}^{K} \mathbf{C}_{k}^{H} \mathbf{B} \mathbf{C}_{k}$. Finalmente, o erro médio quadrático normalizado (EMQN) associado ao conjunto dos $K$ usuários é dado por $e^{2}=\frac{\epsilon^{2}}{M}$ :

$$
e^{2}=\frac{1}{M N_{p}}\left[\mathrm{E}\left[\mathbf{q}_{1}^{H} \mathbf{D}_{o} \mathbf{q}_{1}\right]-M\right]+\frac{\sigma^{2}}{M N_{p}} \operatorname{tr}(\mathbf{B}) .
$$

Expressando (13) em termos da resposta ao impulso do canal (isto é $\mathbf{q}_{1}=\sqrt{M} \mathbf{F}_{M, G} \mathbf{h}_{1}$ ), obtém-se para o EMQN:

$$
\begin{aligned}
e^{2} & =\frac{1}{N_{p}}\left[\mathrm{E}\left[\mathbf{h}_{1}^{H} \mathbf{F}_{M, G}^{H} \mathbf{D}_{o} \mathbf{F}_{M, G} \mathbf{h}_{1}\right]-1\right]+\frac{\sigma^{2}}{M N_{p}} \operatorname{tr}(\mathbf{B}) \\
& =\frac{1}{N_{p}}\left[\mathrm{E}\left[\mathbf{h}_{1}^{H} \mathbf{D}_{F} \mathbf{h}_{1}\right]-1\right]+\frac{\sigma^{2}}{M N_{p}} \operatorname{tr}(\mathbf{B})
\end{aligned}
$$

onde

$$
\mathbf{D}_{F}=\mathbf{F}_{M, G}^{H} \mathbf{D}_{o} \mathbf{F}_{M, G}=\mathbf{F}_{M, G}^{H}\left(\sum_{k=1}^{K} \mathbf{C}_{k}^{H} \mathbf{B C}_{k}\right) \mathbf{F}_{M, G} .
$$

\section{Limitantes do Desempenho EMQN}

Com o objetivo de obter os limitantes inferior e superior do erro médio quadrático normalizado dado em (14), uma operação de decomposição em autovalores pode ser aplicada para obter o autovalor mínimo $\lambda_{\min }$ e o autovalor máximo $\lambda_{\max }$, respectivamente, da matriz $\mathbf{D}_{F}$ de dimensões $G \times G$. Assim:

$$
\left\|\mathbf{h}_{1}\right\|^{2} \lambda_{\min } \leq \mathbf{h}_{1}^{H} \mathbf{D}_{F} \mathbf{h}_{1} \leq \lambda_{\max }\left\|\mathbf{h}_{1}\right\|^{2} .
$$

Tomando-se o valor esperado em (16) e lembrando que $\mathrm{E}\left[\left\|\mathbf{h}_{1}\right\|^{2}\right]=1$, chega-se a:

$$
\begin{aligned}
\mathrm{E}\left[\left\|\mathbf{h}_{1}\right\|^{2}\right] \lambda_{\min } & \leq \mathrm{E}\left[\mathbf{h}_{1}^{H} \mathbf{D}_{F} \mathbf{h}_{1}\right] \leq \lambda_{\max } \mathrm{E}\left[\left\|\mathbf{h}_{1}\right\|^{2}\right] \\
\lambda_{\min } & \leq \mathrm{E}\left[\mathbf{h}_{1}^{H} \mathbf{D}_{F} \mathbf{h}_{1}\right] \leq \lambda_{\max }
\end{aligned}
$$

Os limitantes inferior e superior do erro médio quadrático normalizado da estimativa de canal em (14), podem ser obtidos através da seguinte expressão:

$$
\frac{1}{N_{p}}\left[\lambda_{\min }-1\right]+\frac{\sigma^{2}}{M N_{p}} \operatorname{tr}(\mathbf{B}) \leq e^{2} \leq \frac{1}{N_{p}}\left[\lambda_{\max }-1\right]+\frac{\sigma^{2}}{M N_{p}} \operatorname{tr}(\mathbf{B}) .
$$

Note que, dadas as $K$ sequências de espalhamento em uso, os limitantes em (18) são gerais e independentes das características do canal. Mais ainda, a formulação acima descrita motiva o desenvolvimento de expressões fáceis de usar para o erro médio quadrático normalizado do estimador convencional $\left(e_{c}^{2}\right)$ e do estimador melhorado $\left(e_{w}^{2}\right)$ descritas adiante. Ainda mais, serão definidos os fatores de redução da IMA $\eta$ e de redução do ruído $\gamma$, que estão presentes no erro médio quadrático normalizado da estimativa do canal, decorrentes da utilização da estimativa melhorada (7) em relação a utilização da estimativa convencional (5). 
1) Caso $\mathbf{A}_{\mathbf{m}}=\mathbf{C}_{m}^{-1}$ : Considerando a estimativa do canal dada em (5) onde $\mathbf{A}_{m}=\mathbf{C}_{m}^{-1}$, as matrizes $\mathbf{B}$ em (11) e $\mathbf{D}_{F}$ em (15) tornam-se:

$$
\mathbf{B}=\frac{1}{K} \sum_{m=1}^{K} \mathbf{C}_{m}^{-1} \mathbf{C}_{m}^{-1 H},
$$

e

$$
\begin{aligned}
\mathbf{D}_{F} & =\mathbf{F}_{M, G}^{H} \sum_{k=1}^{K} \operatorname{diag}\left(\mathbf{c}_{k}^{*}\right) \mathbf{B} \operatorname{diag}\left(\mathbf{c}_{k}\right) \mathbf{F}_{M, G} \\
& =\mathbf{F}_{M, G}^{H}\left(\sum_{k=1}^{K} \mathbf{c}_{k}^{*} \mathbf{c}_{k}^{T} \odot \mathbf{B}\right) \mathbf{F}_{M, G} \\
& =\mathbf{F}_{M, G}^{H}(\mathcal{C} \odot \mathbf{B}) \mathbf{F}_{M, G},
\end{aligned}
$$

onde usou-se o fato de que as matrizes $\mathbf{C}_{k}=\operatorname{diag}\left(\mathbf{c}_{k}\right)$ são diagonais, de dimensões $M \times M$ e, assim, definiu-se a matriz $\mathcal{C}=\sum_{m=1}^{K} \mathbf{c}_{m}^{*} \mathbf{c}_{m}^{T}$; e $\odot$ representa o produto de Hadamard ou ponto-a-ponto. Observa-se que utilizando códigos normalizados é possível chegar às seguintes igualdades, entre as matrizes diagonais dos códigos de espalhamento: $\mathbf{C}_{m}^{-1} \mathbf{C}_{m}^{-1 H}=$ $M \mathbf{I}_{M, M}$ e $\mathbf{C}_{k}^{H} \mathbf{C}_{k}=\frac{1}{M} \mathbf{I}_{M, M}$. Assim, a matriz B em (19) reduz-se a:

$$
\mathbf{B}=\mathbf{B}_{c}=\mathbf{C}_{m}^{-1} \mathbf{C}_{m}^{-1 H}=M \mathbf{I}_{M, M},
$$

e seu traço dado é por:

$$
\operatorname{tr}\left(\mathbf{B}_{c}\right)=M \operatorname{tr}\left(\mathbf{I}_{M, M}\right)=M^{2} .
$$

Por sua vez, a matriz $\mathbf{D}_{F}$ em (20) torna-se:

$$
\mathbf{D}_{F}=\mathbf{D}_{F}^{c}=K \mathbf{F}_{M, G}^{H} \mathbf{F}_{M, G}=K \mathbf{I}_{G, G} .
$$

Resulta, portanto, que neste caso $\lambda_{\max }=\lambda_{\min }=K$. Aplicando-se esses resultados em (18) conclui-se que o erro médio quadrático normalizado da estimativa convencional $\left(e_{c}^{2}\right)$ reduz-se à seguinte expresssão:

$$
e_{c}^{2}=\frac{1}{N_{p}}[K-1]+\frac{M \sigma^{2}}{N_{p}} .
$$

É interessante notar que o erro médio quadrático normalizado da estimativa média do canal em (24), utilizando a estimativa MMSE convencional dada por (5), resulta independente das características estatísticas dos canais dos usuários (supondo que são igualmente distribuídos) e dos códigos utilizados; é dependente, apenas, do número de usuários ativos $K$, do número de subportadoras $M$, do número de símbolos piloto transmitidos $N_{p}$, e da potência do ruído $\sigma^{2}$.

2) Caso $\mathbf{A}_{m}=\mathbf{W}_{o} \mathbf{C}_{m}^{-1}$ : Considerando a estimativa melhorada do canal dada em (7) onde $\mathbf{A}_{m}=\mathbf{W}_{o} \mathbf{C}_{m}^{-1}$, e usando a seguinte propriedade da matriz de projeção $\mathbf{W}_{o}^{H} \mathbf{W}_{o}=$ $\mathbf{W}_{o} \mathbf{W}_{o}^{H}=\mathbf{W}_{o}$, a matriz $\mathbf{B}$ em (11) torna-se:

$$
\begin{aligned}
\mathbf{B}=\mathbf{B}_{w} & =\frac{1}{K} \sum_{m=1}^{K} \mathbf{C}_{m}^{-1} \mathbf{W}_{o} \mathbf{C}_{m}^{-1 H} \\
& =\frac{1}{K} \sum_{m=1}^{K} \operatorname{diag}\left(\mathbf{c}_{m}^{\prime}\right) \mathbf{W}_{o} \operatorname{diag}\left(\mathbf{c}_{m}^{\prime *}\right) \\
& =\frac{1}{K} \sum_{m=1}^{K} \mathbf{c}_{m}^{\prime} \mathbf{c}_{m}^{\prime *} \odot \mathbf{W}_{o}=\frac{1}{K}\left(\mathcal{C}^{\prime} \odot \mathbf{W}_{o}\right)(25)
\end{aligned}
$$

com a matriz $\mathcal{C}^{\prime}=\sum_{m=1}^{K} \mathbf{c}_{m}^{\prime *} \mathbf{c}_{m}^{\prime}$, e na qual $\mathbf{c}_{k}^{\prime}$ representa o vetor cujas componentes são os inversos dos elementos do código $\mathbf{c}_{k}$ associado ao $k$-ésimo usuário. Sabendo que $\operatorname{tr}\left(\mathbf{W}_{o}\right)=\frac{G}{M}$ tem-se que:

$$
\operatorname{tr}\left(\mathbf{B}_{w}\right)=\frac{1}{K} \operatorname{tr}\left(\mathcal{C}^{\prime} \odot \mathbf{W}_{o}\right)=\frac{1}{K} K M \operatorname{tr}\left[\mathbf{W}_{o}\right]=M \frac{G}{M}=G .
$$

A matriz $\mathbf{D}_{F}$ em (15) torna-se:

$$
\begin{aligned}
\mathbf{D}_{F}=\mathbf{D}_{F}^{w} & =\mathbf{F}_{M, G}^{H} \sum_{k=1}^{K} \mathbf{C}_{k}^{H} \mathbf{B}_{w} \mathbf{C}_{k} \mathbf{F}_{M, G} \\
& =\mathbf{F}_{M, G}^{H} \sum_{k=1}^{K} \mathbf{C}_{k}^{H}\left(\frac{1}{K}\left(\mathcal{C}^{\prime} \odot \mathbf{W}_{o}\right)\right) \mathbf{C}_{k} \mathbf{F}_{M, G} \\
& =\frac{1}{K} \mathbf{F}_{M, G}^{H}\left(\mathcal{C} \odot \mathcal{C}^{\prime} \odot \mathbf{W}_{o}\right) \mathbf{F}_{M, G} \cdot
\end{aligned}
$$

Uma vez definidas as expressões em (25), (26) e (27) é possível expressar o EMQN melhorado $\left(e_{w}^{2}\right)$ no qual utilizouse a estimativa dada em (7):

$$
e_{w}^{2}=\frac{1}{N_{p}}\left[\mathrm{E}\left[\mathbf{h}_{1}^{H} \mathbf{D}_{F}^{w} \mathbf{h}_{1}\right]-1\right]+\frac{G \sigma^{2}}{N_{p}} .
$$

Os limitantes inferior e superior do erro médio quadrático normalizado melhorado (28) são dados por:

$$
\frac{1}{N_{p}}\left[\lambda_{\min }-1\right]+\frac{G \sigma^{2}}{N_{p}} \leq e_{w}^{2} \leq \frac{1}{N_{p}}\left[\lambda_{\max }-1\right]+\frac{G \sigma^{2}}{N_{p}} .
$$

A comparação de (24) e (28) motiva a definição do fator de redução da interferência de múltiplo acesso (IMA) como:

$$
\eta=\frac{1}{K-1} \mathrm{E}\left[\left[\mathbf{h}_{1}^{H} \mathbf{D}_{F}^{w} \mathbf{h}_{1}\right]-1\right]
$$

bem como o fator de redução do ruído

$$
\gamma=\frac{\operatorname{tr}\left(\mathbf{B}_{w}\right)}{\operatorname{tr}(\mathbf{B})}=\frac{G}{M}
$$

Em (31), $\gamma=\frac{G}{M}<1$, o que evidencia a redução do ruído. Escrevendo (29) em termos do fator de redução da IMA, podem ser obtidos os limitantes inferior e superior do erro médio quadrático normalizado melhorado $e_{w}^{2}$ em função de $\eta$ :

$$
\frac{K-1}{N_{p}} \eta_{\min }+\frac{G \sigma^{2}}{N_{p}} \leq e_{w}^{2} \leq \frac{K-1}{N_{p}} \eta_{\max }+\frac{G \sigma^{2}}{N_{p}},
$$

onde $\eta_{\min }$ e $\eta_{\max }$ podem ser obtidos, respectivamente, através das seguintes expressões:

$$
\eta_{\min }=\frac{\lambda_{\min }-1}{K-1}, \quad \text { e } \quad \eta_{\max }=\frac{\lambda_{\max }-1}{K-1} .
$$

Ressalta-se novamente em (32) que os limitantes são gerais e independentes das características do canal (dependendo entretanto da escolha dos códigos dos usuários). Observando as equações (30) e (31) pode ser verificado que a melhoria no desempenho é diferente para a componente da IMA e para a componente do ruído aditivo presentes no erro da estimativa do canal. 


\section{Um Caso Particular: Perfil Multipercurso do Canal}

Sejam os coeficientes do canal de transmissão do $k$-ésimo usuário dados por $h_{k_{i}}=\alpha_{k_{i}} p_{i} ; i=0, \cdots, L-1$ onde $\alpha_{k_{i}}$ são variáveis aleatórias complexas descorrelatadas com média zero e $\mathrm{E}\left[\left|\alpha_{k_{i}}\right|^{2}\right]=1$; os pesos $p_{i}$ satisfazem $\sum_{i=1}^{L}\left|p_{i}\right|^{2}=1$ e são comuns para os $K$ usuários. Assim, para o estimador de canal melhorado (i.e. caso $\mathbf{A}_{m}=\mathbf{W}_{0} \mathbf{C}_{m}^{-1}$ ), tem-se:

$$
\eta=\frac{1}{K-1} \sum_{i=1}^{L}\left|p_{i}\right|^{2}\left(d_{i, i}^{w}-1\right),
$$

onde $\left\{d_{i, j}^{w}\right\}$ são elementos da matriz $\mathbf{D}_{F}^{w}$ em (27). O EMQN do estimador de canal melhorado, também, pode ser expresso em termos do perfil multipercurso do canal:

$$
e_{w}^{2}=\frac{1}{N_{p}} \sum_{i=1}^{L}\left|p_{i}\right|^{2}\left(d_{i, i}^{w}-1\right)+\frac{\sigma^{2} G}{N_{p}} .
$$

\section{EQuAlizaÇÃo E DETECÇÃo}

Usando os estimadores de canal obtidos, a equalização de (1) seguida da detecção do símbolo pode ser realizada. Neste estudo foi utilizado o equalizador MMSE que tem como solução $\mathbf{w}_{m}=\mathbf{R}^{-1} \mathrm{E}\left[\mathbf{r}(i) b_{m}^{*}\right]$, onde a matriz $\mathbf{R}^{-1}$ é a inversa da matriz de autocorrelação do vetor $\mathbf{r}(i)$ em (1). A matriz de autocorrelação é estimada usando $\widehat{\mathbf{R}}(i)=$ $\frac{1}{i} \sum_{j=1}^{i} \mathbf{r}(j) \mathbf{r}^{H}(j)$. Tendo em vista que $\mathbf{C}_{m}^{-1} \mathbf{E}\left[\mathbf{r}(i) b_{m}^{*}(i)\right]=$ $\mathbf{q}_{m}$, o termo $\mathbf{E}\left[\mathbf{r}(i) b_{m}^{*}(i)\right]$ pode ser aproximado por $\mathbf{C}_{m} \widehat{\mathbf{q}}_{m}$ onde a estimativa $\widehat{\mathbf{q}}_{m}$ pode ser a convencional dada em (5), ou a estimativa de canal melhorada dada em (7). Uma estimativa do símbolo $b_{m}(i)$ transmitido pelo $m$-ésimo usuário pode ser obtida através de $\widehat{b}_{m}(i)=\operatorname{sgn}\left[\operatorname{Re}\left(\mathbf{w}_{m}^{H} \mathbf{r}(i)\right)\right]$ no caso de modulação BPSK.

\section{RESUlTADOS EXPERIMENTAIS}

Os resultados apresentados consideram o sistema MCCDMA síncrono com modulação BPSK. Os $K$ canais são modelados como filtros FIR, cada um com $L=6$ coeficientes invariantes no tempo. O intervalo de guarda é $G=8$ para garantir a detecção livre de IES. A Figura 1 ilustra o EMQN $e_{c}^{2}$ da estimativa convencional, apresentado em (24); os limitantes inferior e superior do EMQN melhorado $e_{w}^{2}$ dados em (32); e o fator de redução do ruído $\gamma=G / M$ versus número de usuários ativos no sistema. No cenário da simulação considerou-se $M=32$ subportadoras, $K=16$ usuários, $N_{p}=128$ símbolos piloto, e $E_{b} / N_{0}=16 \mathrm{~dB}$. A simulação inicia com a seleção aleatória de um grupo de $K$ assinaturas obtido a partir de um conjunto de $M=32$ seqüências Walsh-Hadamard disponíveis. As equações (24) e (32) foram computadas para cada grupo. A seguir um novo grupo foi aleatoriamente selecionado e o procedimento foi repetido. Os resultados ilustrados correspondem à média dos valores do limitante inferior e do superior obtidos em 100 seleções de grupos com $K$ usuários. Note que os limitantes de $e_{w}^{2}$ são bastante próximos. Ademais, verifica-se que $e_{w}^{2}<e_{c}^{2}$, ou seja, o estimador é, realmente, um estimador melhorado. Além disso, observa-se que a utilização do estimador melhorado na estimação de canal permite a redução da componente relativa

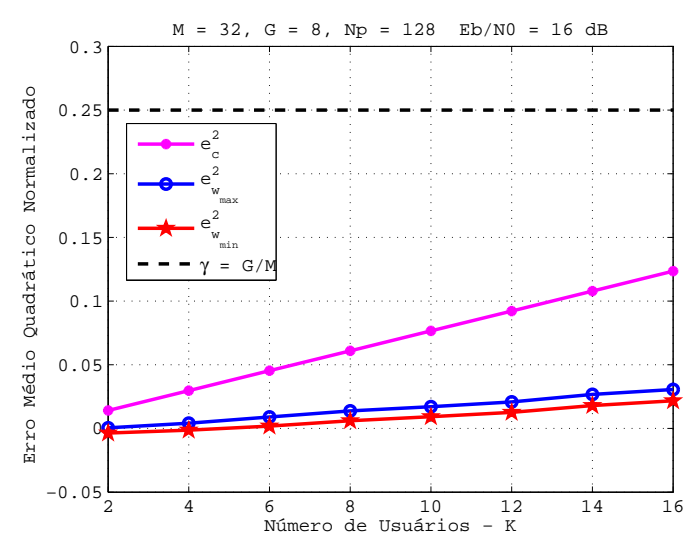

Fig. 1. Limitantes do Erro Médio Quadrático Normalizado Melhorado e Convencional e Fator de Melhoria do Ruído

ao ruído aditivo no EMQN do estimador convencional de $25 \%$ do seu valor original. Na Figura 2 estão ilustrados os limitantes inferior e superior do fator de redução da IMA $\eta$. O mesmo procedimento sobre os possíveis grupos com $K$ assinaturas descrito para a figura anterior, também, foi usado neste teste. Desde que $\eta_{\min }$ e $\eta_{\max }$ em (33) são menores que a unidade, é possível reduzir a componente da IMA presente no EMQN. Além disso, nota-se que os valores de $\eta$ convergem para um valor abaixo do patamar exibido pelo fator de redução do ruído $\gamma=G / M=0,25$. Indicando, assim que na média a redução na componente da IMA é mais significativa que a redução na componente do ruído. Quando o sistema tende a operar com carga máxima os valores de $\eta$ convergem para o nível de $\eta=$ $\frac{G-1}{M-1}=0,226$ que é menor que o valor do fator de redução do ruído $\gamma=0,25$. Considerando agora o perfil multipercurso

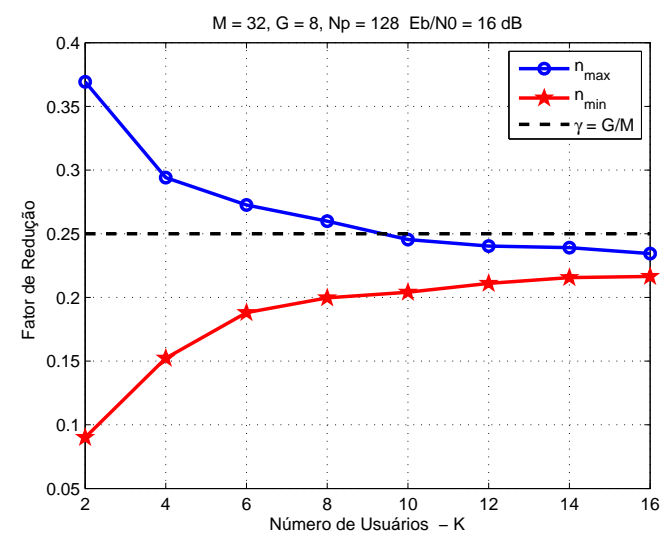

Fig. 2. Limitantes do Fator de Redução da Parcela da IMA e Fator de Redução da Parcela do Ruido

do canal anteriormente descrito, os coeficientes invariantes no tempo são dados por $h_{k_{i}}=p_{i} \alpha_{k_{i}} ; i=0, \cdots, L-1$ onde $\alpha_{k_{i}}$ é uma variável aleatória gaussiana complexa com média nula e $\mathrm{E}\left[\left|\alpha_{k_{i}}\right|^{2}\right]=1$. Os valores de $\alpha_{k_{i}}$ são sorteados aleatoriamente e mantidos fixos ao longo de cada experimento. Os pesos $p_{i}$ satisfazem $\sum_{i=0}^{L-1}\left|p_{i}\right|^{2}=1$. Os valores escolhidos são: $p_{0}=0.74, p_{1}=-0.42, p_{2}=0.083, p_{3}=0.49$, $p_{4}=-0.12, p_{5}=0.01$ [10]. Na Figura 3 estão ilustrados os 
desempenhos médios simulados do EMQN dos estimadores de canal apresentados que são comparados com as expressões teóricas do estimador convencional e do estimador melhorado dadas, respectivamente, em (24) e (35). Os resultados são a média de 100 realizações, e em cada uma delas $N_{B}=2500$ blocos de $M$ símbolos foram considerados. Os resultados simulados e teóricos são concidentes em toda a faixa de valores e o estimador de canal melhorado é menos sensível ao aumento de carga do sistema. Com o objetivo de avaliar o
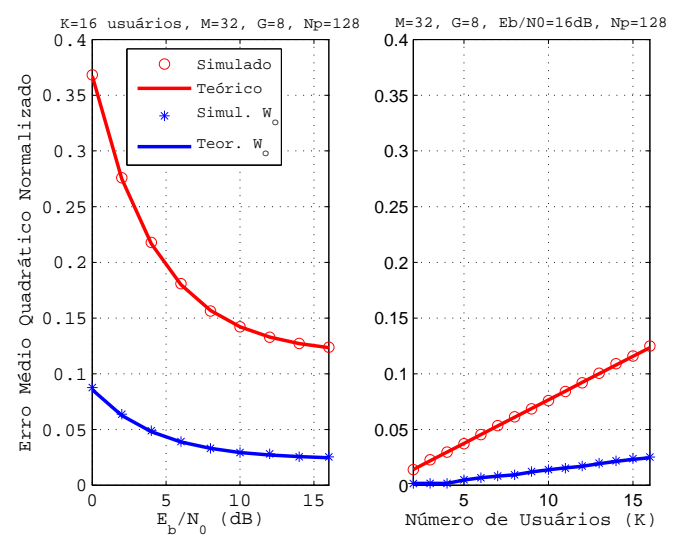

Fig. 3. Desempenho do Erro Médio Quadrático Normalizado versus (a) $E_{b} / N_{0}$ (b) Número de Usuários (K) - MC-CDMA-CP - Enlace Reverso

impacto dos estimadores propostos no desempenho do sistema, na Figura 4 estão ilustradas as curvas de desempenho da taxa de erro de bit em presença de ruído branco gaussiano em dois cenários distintos do sistema MC-CDMA-CP operando com carga máxima. No primeiro cenário $K=M=16$ e no segundo cenário $K=M=32$, em ambos foram utilizados 128 símbolos piloto, intervalo de guarda $G=6$ e equalização MMSE. Na simulação utilizou-se 2500 símbolos e 100 testes foram executados. Observa-se que o desempenho do sistema, com carga máxima, utilizando a estimativa de canal melhorada apresenta significativa redução na taxa de erro de bit, principalmente, em ambientes de alta razão sinal ruído.

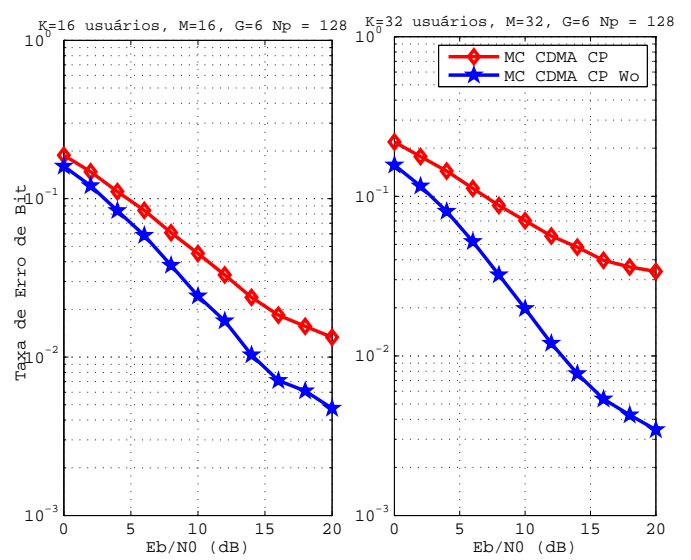

Fig. 4. Desempenho da Taxa de Erro de Bit versus $E_{b} / N_{o}(\mathrm{~dB})$

\section{Conclusões}

Os resultados numéricos mostraram que o procedimento simples para melhoria de estimadores da resposta de frequência do canal resulta em uma redução significativa em ambas as componentes, IMA e ruído, presentes no erro médio quadrático normalizado dos estimadores. Os resultados obtidos por simulação para um receptor MMSE indicaram ganho considerável no desempenho em termos de taxa de erro de bit do sistema. Por fim, é importante observar que as expressões analíticas derivadas podem também ser usadas no projeto de um sistema para selecionar, a partir de um determinado conjunto de códigos de espalhamento, o subconjunto ótimo para ser designado aos diversos usuários do sistema.

\section{APÊNDICE I}

Este apêndice mostra a redução no erro da estimação resultante do uso da matriz de projeção $\mathbf{W}_{o}$. Seja $\widehat{\mathbf{q}}$ uma estimativa de um vetor $\mathbf{q} \in \mathrm{C}^{M}, \widehat{\mathbf{q}}=\mathbf{q}+(\widehat{\mathbf{q}}-\mathbf{q})=\mathbf{q}+\varepsilon$. Suponha que o vetor $\mathbf{q}$ é $\mathbf{q}=\mathbf{F h}$, i.e, $\mathbf{q}$ está contido no subespaço gerado pelas colunas da matriz $\mathbf{F}$ (com dimensão $L \leq M$ ). Seja $\mathbf{W}_{o}$ a matriz que projeta o vetor $\widehat{\mathbf{q}} \in \mathrm{C}^{M}$ nesse subspaço: $\mathbf{W}_{0}=\mathbf{F}\left(\mathbf{F}^{H} \mathbf{F}\right)^{-1} \mathbf{F}^{H}$. Então $\widehat{\mathbf{q}}_{p}=\mathbf{W}_{0} \widehat{\mathbf{q}}=\mathbf{W}_{0} \mathbf{q}+\mathbf{W}_{0} \varepsilon=$ $\mathbf{q}+\varepsilon_{p}$ onde $\varepsilon_{p}$ é a projeção do vetor erro $\varepsilon$ no subspaço gerado por $\mathbf{F}$. Uma vez que $\varepsilon \in \mathrm{C}^{M}$, pode-se escrever $\varepsilon=\mathbf{W}_{o}^{\perp} \varepsilon+\mathbf{W}_{o} \varepsilon=\mathbf{W}_{o}^{\perp} \varepsilon+\varepsilon_{p}$ onde $\mathbf{W}_{o}^{\perp}=\left(\mathbf{I}-\mathbf{W}_{o}\right)$. Então, tem-se: $\|\varepsilon\|^{2}=\left\|\mathbf{W}_{o}^{\perp} \varepsilon\right\|^{2}+\left\|\varepsilon_{p}\right\|^{2}+2 \operatorname{Re}\left[\varepsilon^{H} \mathbf{W}_{o}^{H} \mathbf{W}_{o}^{\perp} \varepsilon\right]$. Como $\mathbf{W}_{o}$ é uma matriz de projeção $\mathbf{W}_{o}^{H}=\mathbf{W}_{o}$ e $\mathbf{W}_{o}^{2}=\mathbf{W}_{o}$, resulta que $\mathbf{W}_{o}^{H} \mathbf{W}_{o}^{\perp}=\mathbf{0}$. Portanto, $\left\|\varepsilon_{p}\right\|^{2} \leq\|\varepsilon\|^{2}$.

\section{REFERÊNCIAS}

[1] K.S.Guilhousen, I.M.Jacobs, R.Padovani, A.J.Viterbi, A.Weaver and C.E.Wheatley. "On the Capaciry of a Celular CDMA System", IEEE Transactions Vehicular Technology,May, v.40, pp. 303-311, 1991.

[2] S. B. Weinstein e P. M, Ebert, Data Transmission by frequency division multiplexing using the discret fourier transform, IEEE Transactions On Communications Tech., COM-19(5), pp. 628-634, Outubro, 1971.

[3] S. Kondo e L. B. Milstein, On the use of multicarrier direct sequence spread spectrum systems, Proc. IEEE Military Communications Conference, MILCOM, pp. 52-56, Outubro, 1993.

[4] B. Muquet, Z. Wang, G. B. Giannakis, M. de Courville and P. Duhamel, "Cyclic Prefixing or Zero Padding for Wireless Multicarrier Transmissions?,'IEEE Trans. Commun., vol. 12, no. 50, p. 2136-2148, 2002.

[5] A. Scherb and V. Kuehn and K.-D. Kammeyer, "Pilot Aided Channel Estimation for short code DS-CDMA", Proc. ISSSTA,pp.39-43, 2002.

[6] J. Van de Beek. O. Edfors, M. Sandell and S. Wilson and P.Börjesson, "On channel estimation for OFDM systems", Transactions on Vehicular Technology Communication, pp. 815-819, Jul., 1995.

[7] R. P. David, "Técnicas de Estimação de Canal Utilizando Símbolos Piloto em Sistemas OFDM", Dissertação de Mestrado - Engenharia Elétrica - PUC-Rio, 2007.

[8] D. F. Cardoso and F. D. Backx and R. Sampaio-Neto, "Performance of Multicarrier CDMA Systems with Improved Pilot-Aided Channel Estimation", WONS - 2009, Fev., Snowbird, EUA, 2009.

[9] D. F. Cardoso and F. D. Backx and R. Sampaio-Neto, "Improved Pilot-Aided Channel Estimation in Zero Padded MC-CDMA Systems", ISWPC - 2009, Fev., Melbourne, Australia, 2009.

[10] H. Sari and G. Karam and I. Jeanclaude, Transmission Techniques for Digital Terrestrial TV Broadcasting, IEEE Communications Magazine, 0163-6804/95, pp. 100-109, 1995. 